\title{
EigenEvent: An algorithm for event detection from complex data streams in syndromic surveillance
}

\author{
Hadi Fanaee-T* and João Gama \\ Laboratory of Artificial Intelligence and Decision Support, University of Porto, Porto, Portugal
}

\begin{abstract}
Syndromic surveillance systems continuously monitor multiple pre-diagnostic daily streams of indicators from different regions with the aim of early detection of disease outbreaks. The main objective of these systems is to detect outbreaks hours or days before the clinical and laboratory confirmation. The type of data that is being generated via these systems is usually multivariate and seasonal with spatial and temporal dimensions. The algorithm What's Strange About Recent Events (WSARE) is the state-of-the-art method for such problems. It exhaustively searches for contrast sets in the multivariate data and signals an alarm when find statistically significant rules. This bottom-up approach presents a much lower detection delay comparing the existing top-down approaches. However, WSARE is very sensitive to the small-scale changes and subsequently comes with a relatively high rate of false alarms. We propose a new approach called EigenEvent that is neither fully top-down nor bottom-up. In this method, we instead of top-down or bottom-up search, track changes in data correlation structure via eigenspace techniques. This new methodology enables us to detect both overall changes (via eigenvalue) and dimension-level changes (via eigenvectors). Experimental results on hundred sets of benchmark data reveals that EigenEvent presents a better overall performance comparing state-of-the-art, in particular in terms of the false alarm rate.
\end{abstract}

Keywords: Event detection, complex data streams, tensor decomposition, syndromic surveillance

\section{Introduction}

The goal of syndromic surveillance systems is to enable earlier detection of epidemics and a more timely public health response, hours or days before clinical and laboratory confirmation comes out [15]. Two kinds of events are usually required to be detected: man-made events such as bio-terrorist activities like anthrax attacks [13] and natural events such as epidemic diseases like H1N1, avian influenza, SARS, and West Nile Virus, etc. All kinds of events regardless of their type make some changes in the environment. If we somehow manage to identify such changes in the early stages we can save many lives and prevent the potential damages. The early event detection systems are developed for such purposes. In these systems, multiple streams of pre-diagnostic health records [15|38] such as daily counts of doctor/hospital/emergency room visits, over-the-counter medication sales, work/school absences, animal illness or deaths, internet-based health inquiries are being monitored simultaneously to trace the event footprints.

\footnotetext{
${ }^{*}$ Corresponding author: Hadi Fanaee-T, Laboratory of Artificial Intelligence and Decision Support (LIAAD), University of Porto INESC TEC, Rua Dr. Roberto Frias, Porto, Portugal. E-mail: hadi.fanaee@ fe.up.pt.
} 


\begin{tabular}{|c|c|c|c|c|c|c|c|c|}
\hline & Zone\#1 & Zone\#2 & Zone\#3 & Zone\#4 & Zone\#5 & Zone\#6 & Zone\#7 & Zone\#8 \\
\hline V1 & $\mapsto \wedge$ NA & 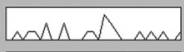 & ranhn & Lumpar & $\square M$ & $M \sim M m$ & $m \wedge M A$ & Nh/ $\mathrm{mm}$ \\
\hline V2 & $M M \sim n \wedge$ & Mundm & thMM & mannand & $\cap \mathrm{Nm}$ & hrmm & Wharw & mandurb \\
\hline V3 & $M \sim m \sim N$ & mancinas & momen & mancmons & $M \sim m$ & hrmans & moversw & MVanm \\
\hline V4 & rown M & mann & momins & mancos & $M A \neg \backslash N^{N}$ & wantrum & Manmond & Jimmons \\
\hline V5 & anshne & mmon & reman & NAnm & $\triangle \wedge \wedge \wedge N^{\prime}$ & Wrowhn & ormanns & $M M \sim n$ \\
\hline V6 & whes & Ansthen & Arman & Munmm & man AmaN & $M M d \wedge M$ & nmmins & $\Lambda$-wnd \\
\hline v7 & Inn & Wr & Mumha & ormans & $M N \neg \wedge \wedge N$ & $n \wedge M N$ & Mnnmants & MNM \\
\hline V8 & Sham $N$ & WWWM & & ardarm & $\Delta \mathrm{mm}$ & havmo & WWW & sthrom \\
\hline V9 & NWMMI & 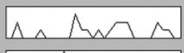 & andmon & Mnh_MNM & $\triangle \wedge \wedge \wedge \wedge$ & $M M \triangle M$ & Mr.Mor & $\Lambda \mathrm{N} \cap \mathrm{MN}$ \\
\hline 10 & man & why & warmad & mammansm & $\Delta$ Am & walluh & $m m \sim v$ & $v^{\prime} n-w$ \\
\hline V11 & & & & & & $A \quad M$ & $\begin{array}{lll} & \wedge & \wedge\end{array}$ & \\
\hline V12 & $\triangle M M \triangle \triangle \sim N$ & Whand MN & then & marnmand & hNר & $1 \mathrm{rrahm}$ & Manmons & 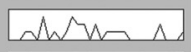 \\
\hline V13 & AlA $\wedge M$ & VMM ${ }^{\wedge}$ & hanemon & $\triangle M M \cap M$ & $\square$ & Lnsus & $\checkmark M \wedge M \sim$ & $M M \sim M$ \\
\hline V14 & Whmen & whMnN & thrownen & AntMarn & Wh $/$ hMn & WN WM & Whar & Mwrlun \\
\hline V15 & $r M \triangle \wedge \triangle A$ & ramun & S-UMw & harmm & An hand & Monnla & whom & Wh and \\
\hline$/ 16$ & & 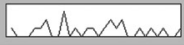 & NMn & 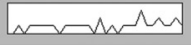 & $\begin{array}{llll}4 & N\end{array}$ & $M M \wedge$ & $M A M n$ & M M MM \\
\hline
\end{tabular}

Fig. 1. A sample complex system in syndromic surveillance that generates 128 time series for 16 features and 8 spatial regions.

Figure 1 demonstrates an example of a complex data stream in synonymic surveillance systems. As it can be seen, this system measures 16 features aggregated daily within 8 different regions. Hence, the system generates 128 time series. Our goal is to monitor this complex system and signal an alarm when something strange occurs. One straightforward approach for monitoring such system is to monitor each individual time series and then apply an anomaly detection technique (e.g. Control chart) on each. This approach, however, imposes much higher false alarm rate. Because pre-diagnostic streams of indicators are weak and noisy signals [4] and applying detectors on each individual signal results in multiple hypothesis testing problem [48]. For instance, suppose that we reject null hypothesis when the $p-$ value $\prec 0.05$, for a single hypothesis test, the probability of making a false discovery is equal to 0.05 . Now assume that we do the test for each of 128 time series. Probability of false alarm could be as bad as: $1-(1-0.05)^{128}=1.00 \gg 0.05$.

Existing univariate methods include statistical process control based approaches [16]46]; Time series analysis and signal processing based approaches, including singular spectrum analysis (SSA) [32], Box-Jenkins models [36]; Wavelet [50], Hidden Markov Model (HMM) [28]35]; and regression [37]. The univariate methods since only monitor a single variable are not proper techniques for handling the complex data in synonymic surveillance. Besides, if we monitor each individual feature independently without taking into account the correlation between them, we then likely confuse the measurements error and noises with the events.

The other category of methods is multivariate methods that are able to monitor multiple streams. These methods include Hotelling T2 [49], multivariate CUSUM and EWMA [30], principal component analysis (PCA) [21], multivariate HMM [31], vector autoregression (VAR) [19] and vector autoregression moving average (VARMA) [8]. There is also a sub-group of multivariate methods that operates on categorical data and looks for interesting rules via contrast set mining techniques. STUCCO [2] and Emerging Patterns [7] are instances of such techniques. Multivariate temporal methods, despite of their wide application in many areas, are not well-suited to syndromic surveillance and outbreak detection problems where geographic dimension is widely involved. 
The methods that take into account geographic dimension are twofold: spatial and spatiotemporal. Spatial methods such as spatial scan statistics [22] do not capture the temporal fluctuations of the data and only operate on spatial data. Spatiotemporal methods instead take into account both spatial and temporal dimensions. Space-time scan statistics (STScan) is of this group that can operate both on univariate count data [22-24|26] and multivariate data [25]. Univariate STScan is not adequate for syndromic surveillance for the same reason mentioned for univariate temporal methods. Multivariate STScan also has some drawbacks that make it be inappropriate for the introduced problem. On one hand they assume that the environment is static and do not consider seasonal effects and on the other hand they are developed for retrospective and offline analysis. Therefore, this group of techniques is not also suited to the problem.

There is another group of techniques such as PANDA [5] that use a causal Bayesian network to model spatiotemporal patterns of outbreaks. These methods not only explicitly compute the probability of events, but also are able to operate in real time settings through incremental updating of the Bayesian network. However, the main criticism against these techniques is that tuning the primary parameters requires a deep prior knowledge that is not available most of the time. Therefore, these methods are considered domain specific and their application has remained limited.

Among many existing techniques and algorithms, the most suited approach to the introduced problem is WSARE [47/48] that is able to handle multivariate data along spatial and temporal dimensions. WSARE searches for surprising rules in data streams given some baseline reference. The baseline creation strategy varies in different version of the algorithm. WSARE 2.0 uses raw historical data from selected days, WSARE 2.5 uses all historical data that match the environmental attributes and WSARE 3.0 models the baseline distribution using a Bayesian network. Opposed to PANDA where Bayesian network is created manually, WSARE 3.0 learns the Bayesian network from historical set. Therefore, is not as such domain-dependent as PANDA. WSARE has been successfully applied and merited in many real world problems such as in bioterrorism surveillance for 2002 Winter Olympics [12] and Israel influenza type B outbreak and Walterton outbreak [18]. However, the main criticism about WSARE is its high rate of false alarms [3]. WSARE opposed to other techniques, processes the data from bottom to up. Therefore, instead of overall changes in the whole data, it tracks the changes in subgroup of data. Therefore, it is sensitive to small changes and consequently presents lower detection delay, however, comes with more false alarm rate.

The methodological differences between our proposed method and WSARE are as follows. 1) WSARE is a bottom-up rule-based approach while our method is a middle approach between bottomup and top-down that tracks both high level and dimension-based changes in the data subspace. 2) Our approach takes into account both multi-linear and multi-way correlations in data while WSARE is not able to capture such complexity; and 3) Our method is suitable only for alarming purposes and cannot explain about subgroup of the data that cause the alarm, while WSARE can be used for both purposes. 4) The statistical significance of the alarms in WSARE is computed via Monte Carlo simulation while in our approach is computed by statistical process control techniques.

In overall, the main objective in syndromic surveillance systems is to detect events in a timely manner before they turn into an epidemic. This early detection has important functions in both mortality saving and prevention of economic losses. An estimation by DARPA shows that a two-day improvement in detection time could reduce fatalities by a factor of six [33]. Another study states that improvements of even an hour in detection can reduce the economic impact of by a hundred million of dollars [34]. To reach this objective, any capable signal is required to be considered. However, this is somehow problematic, since involving more signals results in more false alarms. In the recent years the emphasis of the developed algorithms in syndromic surveillance has been focused more on the early detection and rate of false alarm is rarely taken into account. This is while the recent studies show that the false alarm 


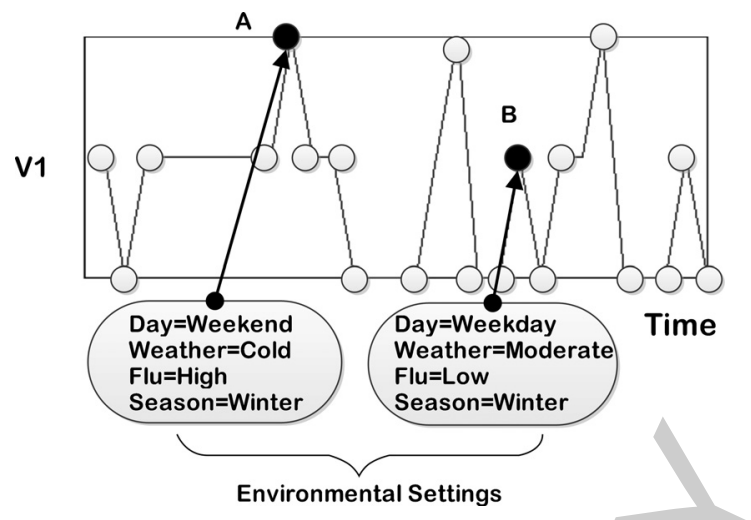

Fig. 2. The environmental setting affects the data items.

rate can have an inverse effect as bad as delay in detection. A recent study concerning the warning system for tornado events [41] reveals that tornadoes occurring in the regions with a high false alarms ratio kill and injure more people. A statistically significant effect of false alarms is identified in this study: A one-standard-deviation increase in the false alarm ratio increases expected fatalities by between $12 \%$ and $29 \%$ and increases expected injuries by between $14 \%$ and $32 \%$.

Besides, opposed to anomaly or outlier detection problems, which it is assumed that the process occurs in an isolated and static environment in synonymic surveillance systems we deal with dynamic and time-changing environment. In such environments, attributes such as day of the week, holiday, weather, etc. affect the whole or part of the system behavior. Figure 2 illustrates an individual time series corresponding to the feature $V 1$ and Zone 1 in Fig. 1 . As it can be seen, in point $A$ due to cold weather and high rate of influenza rate, we have a higher count comparing point B. Such effects impose another kind of complexity to the event detection problem in syndromic surveillance which is required to be taken into account along with other issues.

In this paper, we propose a novel event detection methodology that considers both data complexity and time-changing environmental issues in syndromic surveillance. The concentration of this work is to reduce the false alarm rate of early event detection systems. Our contributions are as follows.

- To the best of our knowledge this is the first time that the tensor decomposition techniques [20] is applied to the syndromic surveillance problem with space and time dimensions.

- We use the changes in data dimensions and data correlation structure as an effective criteria for event detection.

- We introduce a novel and effective approach for baseline data creation that can infer baseline for unseen environmental settings.

The rest of the paper is organized as follows. In Section 2 we introduce the proposed solution and our developed algorithm EigenEvent. The Section 3 includes experimental evaluation, including the introduction of the data set, performance evaluation and sensitivity analysis. The last section concludes the exposition presenting the final remarks.

\section{Proposed method}

\subsection{The idea}

The fundamental idea that is used to develop the method relies on tracking changes in the subspace. 

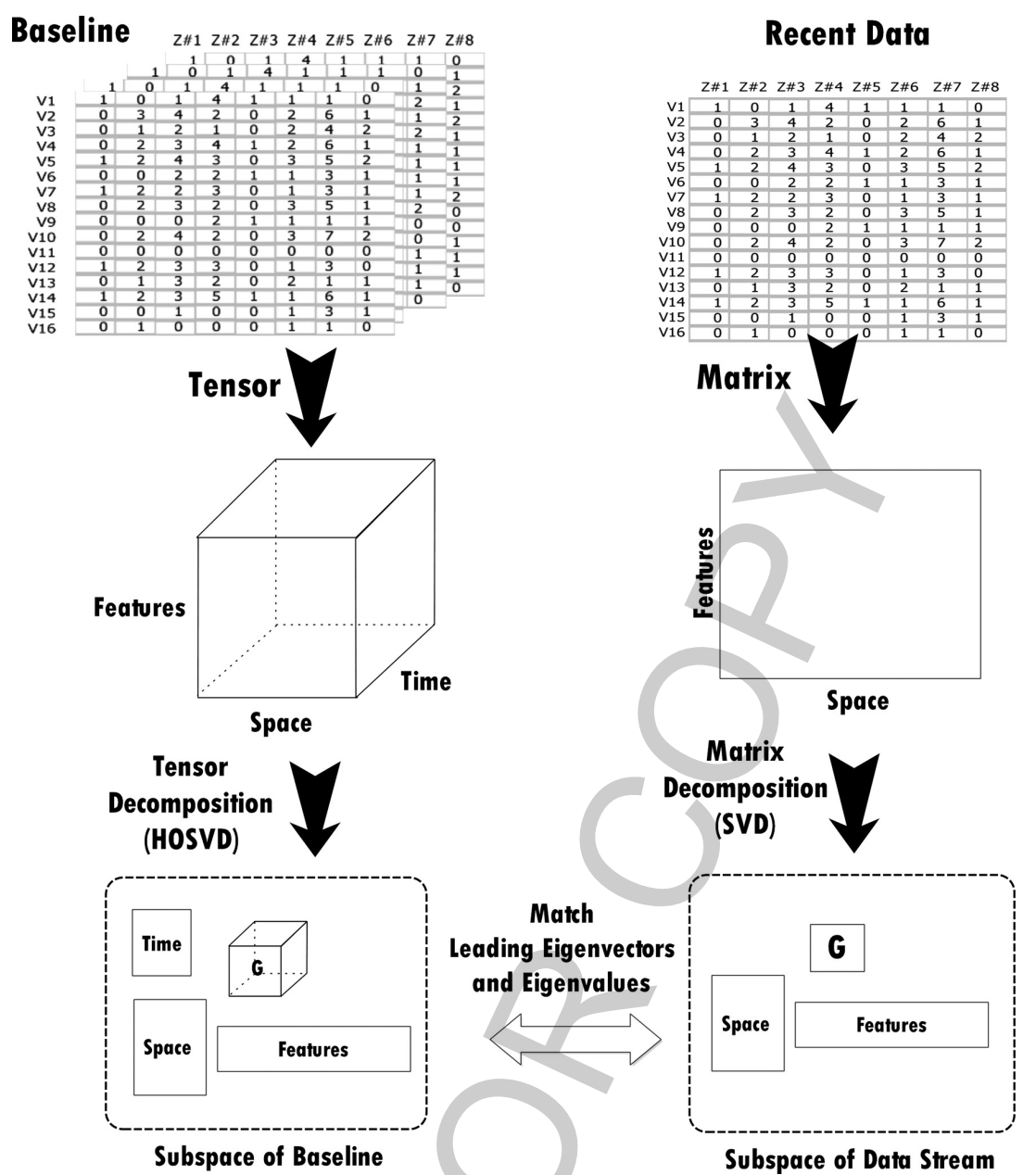

Fig. 3. Snapshot of the proposed solution at a hypothetical timestamp. We detect events through tracking changes in the subspaces of baseline and recent data.

This is impossible unless we could match the recent data with a baseline reference. However, in streaming settings, data itself is time-changing due to the effect of the dynamic environment on the data items. Therefore, using a static baseline seems to be inappropriate for dynamic environments. We propose a dynamic baseline set creation strategy which takes into account both seasonality and non-stationarity. The main novelty of our method is that we not only track changes in the feature subspace, but in the subspace of other dimensions.

Figure 3 demonstrates an illustrative example of our proposed method. Each day we receive a chunk from a complex data stream. In the data stream model this can be translated to the sliding window with fixed size of one day across the data stream. The window here is more complicated than a onedimensional window in temporal data processing. Each window is a two-dimensional matrix of Space $\times$ Features (top-right matrix). Each cell in the matrix corresponds to the count of a feature in specific regions. With respect to the sliding window environmental setting, we generate a dynamic baseline tensor with order of Space $\times$ Features $\times$ Time (top-left tensor) which is being fed from the historical data. This baseline tensor is built in each step or cycle of the algorithm run. The baseline tensor is composed 


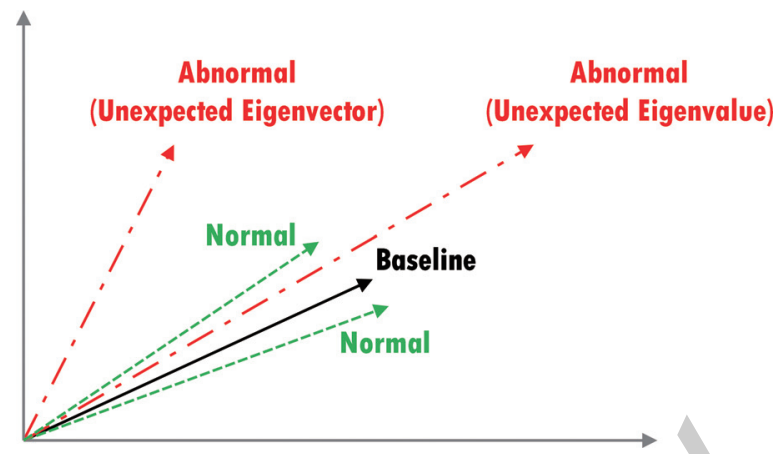

Fig. 4. A simplified example showing how events can be detected by tracking changes in the eigenspace. If the distance between the sliding window eigenvector and baseline eigenvector is higher than expected, then the window is marked as abnormal. Also, if the ratio of window eigenvalue to the baseline eigenvalue is higher than expected, the window is marked as abnormal as well. (Colours are visible in the online version of the article; http://dx.doi.org/10.3233/IDA-150734)

of some previously arrived sliding windows that are combined in a particular order. We decompose the recent matrix and the baseline tensor to a lower-rank subspace and then match their pairwise eigenvectors and eigenvalues. We signal an alarm if we observe any unexpected difference in the match.

Figure 4 illustrates the eigenspace of both baseline and recent matrix. The solid vector in this figure corresponds to the baseline tensor. The direction of this vector corresponds to the principal eigenvector corresponding to a dimension and the length of the vector corresponds to the principal eigenvalue. When we receive a matrix we decompose it to the eigenvectors and eigenvalues and then match the obtained principal eigenvalue and principal eigenvectors to the reference vector (solid vector). We signal an alarm if the matrix eigenvector has a considerable difference in direction (eigenvector) or length (eigenvalue). For instance, dashed lines in the figure correspond to those matrices that have close eigenvector to the baseline eigenvector and have the close eigenvalue (vector length). Such matrices are considered normal by EigenEvent. Dash-dot lines in the figure on the contrary are related to abnormal matrices that have an unexpected eigenvector (unexpected vector direction) or unexpected eigenvalue (unexpected vector length) with respect to the baseline.

\subsection{Proposed algorithm: EigenEvent}

In this section we describe our proposed algorithm, which is called EigenEvent. As it is presented in Algorithm 1, the inputs are as follows: sliding window $D$ with length of one day; $t$ which is the sequence number; $e$ is a number corresponding to the environmental setting of the day. For instance, the environmental setting 1214 is related to: day $=$ weekend (1), weather $=$ cold (2), flu $=$ high (1), season $=$ winter (4). The algorithm as a result outputs a $p$-value indicating the statistical significance of the sliding window. A very low $p$-value can be interpreted as an event signal.

\subsubsection{Data processing and decomposition}

The first phase is to transform the sliding window to the matrix format of Space $\times$ Feature (line 1). To assess the abnormality of sliding window we need a baseline reference to match with. Two strategies can be utilized, one is to compare the window with the previous data and another strategy is to compare the window with previous data that have the same environmental setting. We use a combined strategy that takes into account both (see Section 2.2.4) and produce the dynamic baseline set according the context corresponding to the window (line 2). As a result, baseline is presented as a tensor of Space $\times$ Feature 


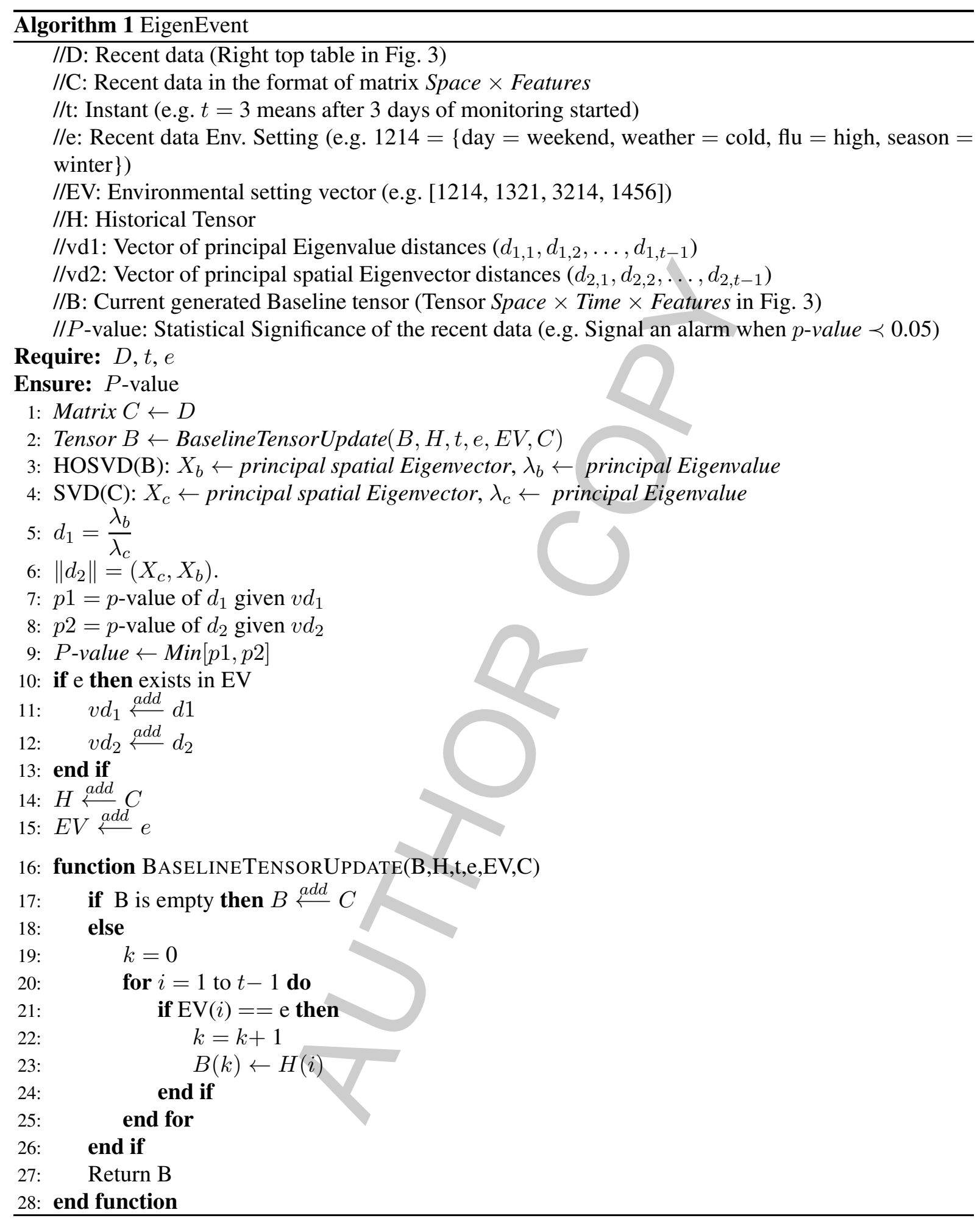


$\times$ Time. We then apply SVD [19] on window matrix and higher order SVD (HOSVD) [6]20] on the baseline tensor and for each dimension we take the principal eigenvector and eigenvalue.

Note that EigenEvent does not concern about the feature selection (selection of pre-diagnostic signals that are required to be monitored). Feature selection, however, may be performed via standard feature selection techniques or via domain experts or a combined technique. Nevertheless, feature selection is one the most important steps in a data mining process that is required to be taken into account. Selection of inappropriate signals may result in higher false alarm or more detection delay. The wellknown over-fitting problem may happen here as well. Leinweber in an article entitle stupid data miner tricks: overfitting the $S \& P 500$ names some of such problems. He finds a strong correlation between butter production in Bangladesh and S\&P 500 (stock market index) over a ten year period. This implies that the selection of appropriate signals still is human-dependent and cannot be fully automated.

\subsubsection{Subspace matching}

The next phase is the matching phase. If we denote the principal eigenvalue of baseline with $\lambda_{b}$, the principal eigenvalue of window with $\lambda_{s}$, the principal eigenvector of baseline with $X_{b}$ and the principal eigenvector of window with $X_{s}$, we can define the ratio of eigenvalues and Euclidean distance of eigenvectors respectively as:

$$
\begin{aligned}
d_{1, t} & =\frac{\lambda_{s}}{\lambda_{b}} \\
\left\|d_{2, t}\right\| & =\left(X_{s}, X_{b}\right) .
\end{aligned}
$$

We keep the historical distances in two vectors of $v d_{1}$ and $v d_{2}$ for eigenvalues and eigenvectors respectively, such that at time $t$ we have $v d_{1}=\left(d_{1,1}, d_{1,2}, \ldots, d_{1, t-1}\right)$ and $v d_{2}=\left(d_{2,1}, d_{2,2}, \ldots, d_{2, t-1}\right)$. Having $d_{1, t}, d_{2, t}, v d_{1}$ and $v d_{2}$ we can compute the $z$-scores corresponding $d_{1, t}$ and $d_{2, t}$ as follows.

$$
\begin{aligned}
& z 1=\frac{d_{1, t}-\mu_{v d_{1}}}{\sigma_{v d_{1}}} \\
& z 2=\frac{d_{2, t}-\mu_{v d_{2}}}{\sigma_{v d_{2}}}
\end{aligned}
$$

Where $\mu_{v d_{1}}$ and $\mu_{v d_{2}}$ denotes the mean and $\sigma_{v d_{1}}$ and $\sigma_{v d_{2}}$ denote standard deviation of vector $v d_{1}$ and $v d_{2}$ respectively.

Although $z$-scores alone can be used along with a threshold for alarming purpose, since most related event detection algorithms in the literature outputs $p$-value, we may want to transform $z$-scores to the corresponding $p$-value to ease the comparison task. We can use the following equation to derive the $p$-value from the $z$-score:

$$
P(z)=\frac{1}{\sqrt{2} \pi} \int_{-\infty}^{z} e^{\frac{-t^{2}}{2}} d t
$$

\subsubsection{Indicator selection}

As we already explained, HOSVD and SVD decompose the complex data into smaller subspaces (eigenspace). Tensor and matrix decomposition methods are robust against the noises. However, in the case that we have some missing values we need to use specific types of SVD [27].

We have three elements in the eigenspace that can be matched: principal eigenvector of spatial and feature dimensions and the principal eigenvalue. We may observe three kinds of changes in the match. 
The first kind includes an overall change in the system which is more related to the late days of outbreak period when we have both infection and outbreak. This kind of event must be reflected in a significant change in the ratio of eigenvalues $\left(d_{1, t}\right)$. The second kind of change occurs when an event agent (e.g. Virus) begins to spread over the geographical space. This type of event also is reflected in the changes in the spatial eigenvector pairwise distance $\left(d_{2, t}\right)$. The third kind is the change in the feature values. This event type can be reflected in the eigenvectors corresponding to feature dimension. However, as we show later due to the noisy properties of the feature dimension, this kind of indicator is not such helpful.

We propose a new strategy that is able to detect both overall and dimension-based changes in the system. We monitor the system using a combination of indicators, including eigenvalue and different eigenvectors and the compute the $p$-value corresponding to each combination for each sliding window. Then we take the minimum $p$-value as the algorithm output (line 9). Suppose that we have three $p$-values of $0.01,0.12$ and 0.43 corresponding to the pairwise match between the principal spatial eigenvector, the principal feature eigenvector and the principal eigenvalue respectively. The EigenEvent algorithm reports the minimum $p$-value $(0.01)$ as the output. These above mentioned $p$-values indicate three facts about the system: 1) No overall change has occurred in the system, because $p$-value corresponding Eigenvalue is considerably high; 2) No significant change is occurring in the feature values; 3) A significant change is occurring in the spatial dimension. We may infer that data items despite of showing normal behavior in the features are showing different behavior in geographical space and hence we probably are in the outbreak phase. The minimum $p$-value selection strategy lets us to detect all above kinds of changes and subsequently makes the algorithm sensitive to changes in both overall system behavior and the dimension level.

\subsubsection{Dynamic baseline tensor}

There should be a criterion to estimate the abnormality of the recent data. As is mentioned before, two types of common criteria includes comparing with the previous data and comparing with only the previous data that match the current environment settings. Both of these criteria are vulnerable. The first criteria fails when data contains seasonal effects and second one fails when there is no enough historical data matching the recent environmental setting. To solve this problem a typical inference usually is performed, for instance, a causal Bayesian network is constructed in WSARE 3.0 [47]48] so that when there is no enough historical data, baseline is inferred from the constructed Bayesian network. This approach, however, only make inference about the days their corresponding environmental settings cannot be found in the baseline set. In the rest of the time it compares the recent window with the previous data that match the current environment settings. This approach can be vulnerable as well, since the correlation of the current window with the recent data is ignored. We introduce another way of baseline set selection which is a combination of both ideas. We assume that the recent data is not only related to the previous data and data with the same environmental settings, but also to data with the most repeated environmental settings. In fact, our baseline tensor is a combination of previous data, data with the same environmental setting and data from most frequent environmental settings. The main advantage of this approach is that it does not fail when deal with an unseen environmental setting.

The function BaselineTensorUpdate in Algorithm 1 receives six inputs, including $B$ (current baseline tensor); $H$ (whole data, historical tensor); $t$ (instant number); $e$ (the recent environmental setting); $E V$ (vector of all environmental settings seen yet); and $C$ (recent matrix) and outputs the updated baseline tensor $B$. It first checks that whether the tensor $B$ is empty. In the case that $B$ is empty, $C$ is added to $B$. Then we search in historical tensor $H$ for data that match the recent environmental setting. Next, it rewrites the first $k$ matrices of tensor $B$ with the matched items.

An illustrative example of the procedure is demonstrated in Fig. 5. The figure is a snapshot of the system at four hypothetical days between days 50 to 53 . From the figure we also can observe four 


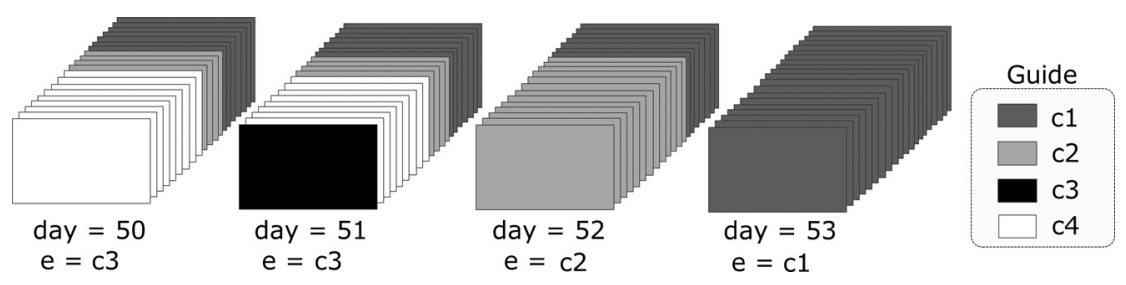

Fig. 5. A Sample of dynamic baseline tensor creation process between day 50 to day 53 . Each plate represents a daily matrix of Space $\times$ Features in historical set. The dynamic baseline tensor is a combination of such matrices in a particular order. $e$ also denotes the environmental setting of the day.

distinct environmental settings, which are shown with different colors and that their corresponding name is demonstrated in the guide table. Each cube in the figure represents a baseline tensor and each plate inside the cubes is a Space $\times$ Features matrix from the historical set. At day 50 the baseline tensor is composed of 20 matrices such that 9 of matrices are from setting $\mathbf{c 4}, 4$ matrices from setting $\mathbf{c 2}$ and 7 matrices from setting c1. We also assume that the context $\mathbf{c 1}$ is the dominant environmental setting with 20 times occurrence. The dominant context is the most frequent setting in all the history. For this reason, all baseline tensors, in Fig. 5, include 20 matrices, given that the length of the baseline tensor is equal to the number of occurrences of the dominant context.

Now let's explain how a dynamic baseline set is generated. At day 50, we receive a matrix with setting c1. We search in historical tensor $H$ for a match with c1 setting, but we do not find, so the function BaselineTensorUpdate returns input $B$ unchanged. On day 51, we again receive a matrix with the setting c1. We again search for a match in $H$. This time we find one match, because one day before (day 50) the setting has been c1. Therefore, we rewrite the first $k$ elements of $B$ Tensor with $k$ found matrices. In this case since we find only one match, $k$ is equal to 1 . At day 52 we receive a matrix corresponding with environmental setting $\mathbf{c 2}$. We search in $\mathrm{H}$ for a match and suppose that we find 13 matrices. Hence, $k$ will be equal to 13 , so we rewrite the first 13 elements of the baseline tensor with the matched 13 matrices. As it can be observed at day 52, setting $\mathbf{c 2}$ has been the dominant setting versus $\mathbf{c 3}$ and $\mathbf{c 4}$ settings, however, still c1 dominates $\mathbf{c 2}$ (c1 setting has more repeats comparing c2), therefore, the baseline tensor is composed of matrices with most dominant settings with preference to the recent data. Finally, on day 53, we receive a matrix with setting c1. We search in $H$ for a match and we find 20 matrices $(k=20)$, thus we rewrite first 20 elements of the baseline tensor with matrices corresponding c1 settings. At this moment, the whole baseline tensor is filled with only matrices with setting c1. This procedure repeats and repeats. However, the size of baseline tensor always stays fixed to the repeat count of the most repeated environmental settings.

\subsubsection{Updating step}

In this step we update the vector of distances (lines 11-12). We add the distances to the vectors if their corresponding contexts has been already seen. If we have a matrix with an unseen environmental setting, we do not add the computed distance to the vector of distances. Because an inference for this setting is approximate and adding the distance obtained from this approximation is not adequate for keeping. We finally update historical tensor and vector of environmental settings.

\section{Evaluation}

\subsection{Data set}

Validation of event detection algorithms is basically a difficult task due to the type of required data [3, 
Table 1

Characteristic of CityBN data sets

\begin{tabular}{llrll}
\hline Field & Type & Cardinality & Sample record \#1 & Sample record \#2 \\
\hline XY & Spatial & 9 & SW & NE \\
Daynum & Temporal & 730 & 73779 & 74508 \\
Age & Feature & 3 & Senior & Child \\
Gender & Feature & 2 & Female & Male \\
Action & Feature & 3 & Purchase & Evisit \\
Reported symptom & Feature & 4 & Nausea & Respiratory \\
Drug & Feature & 4 & Nyquil & Vomit-b-gone \\
Flu level in season & Environmental & 4 & High & Decline \\
Day of week & Environmental & 3 & Weekday & Sat \\
Weather & Environmental & 2 & Cold & Hot \\
Season & Environmental & 4 & Winter & Sumer \\
\hline
\end{tabular}

39[48]. To evaluate the algorithms, the event occurrence period is required to clearly be labeled in the data. This requires a knowledge expert to look into the data and specify the event period manually, making this task infeasible. Benchmark data sets that are already used for change detection and anomaly detection are not appropriate for our research purpose, because, on one hand, most of the time they do not have seasonality property and on the other hand do not contain multi-way property. We recently [10] proposed a semi-automatic for labeling events in unlabeled data which is based on ensemble detectors and background knowledge from web. However, this approach also needs to have access to some sort of background knowledge which is not available in this domain.

We use a benchmark data set used in [47] including 100 data sets of a simulated disease outbreak. These data sets are generated using a Bayesian network simulator namely CityBN which generates temporal fluctuations based on a variety of factors such as weather and food conditions [48]. The structure and parameter of this Bayesian network are manually adjusted. As is mentioned by the authors, this simulator produces extremely noisy data sets that are a challenge for any detection algorithm. This data set is publicly available online in [45].

Table 1 shows the characteristic of the original data sets. As it can be seen, this data is multi-way. It contains two dimensions of space and time and multiple variables. It also contains seasonal effects, because features are under influence of some environmental settings. Cardinality of each attribute is also specified in the table. As it can be seen, we have 9 distinct spatial regions and 730 temporal instants (days). We also have $16(3+2+3+4+4)$ distinct time series and $4 \times 3 \times 2 \times 4$ possible environmental settings.

\subsection{Performance}

Receiver operating characteristic (ROC) curve [14] measures the trade-off between sensitivity and specificity. ROC curve is widely used method for evaluation of anomaly detection and classification methods. However, ROC curve, even though summarizes the overall ability of the algorithm, does not evaluate the timeliness of detection which is critical in syndromic surveillance. An algorithm with the lowest false positive and the highest true positive rate that detect outbreaks with heavy delay is inappropriate for syndromic surveillance applications. In fact a system with this characteristic is more helpful for retrospective applications than the prospective applications like what is required in syndromic surveillance. One of the proper metrics for evaluation of algorithms is Activity Monitoring Operating Characteristic (AMOC) curve [11] that evaluates the trade-off between specificity (false alarms) and timeliness (detection time). AMOC curve is widely used for evaluation of methods in syndromic surveillance [4[17|40[48]. Therefore, in this work we use AMOC curve for evaluation of our algorithm. 


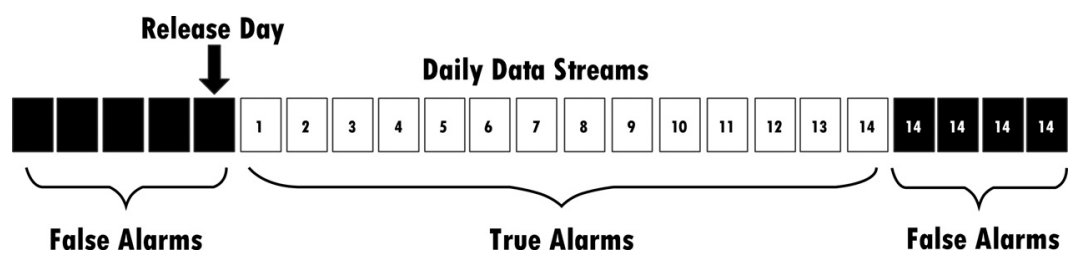

Fig. 6. Evaluation strategy: Alarms in days with black color represents false alarms and in white days represents true alarms. Detection delay is also specified for each day inside the plates.

We use the same evaluation strategy as [48]. Assume that the agent release occurs at timestamp $t$. A true alarm corresponds to a case where the alarm is raised in a period between $t+1$ and $t+14$. The alarms before or after this period are considered false positives. The detection delay is also defined as the temporal difference between the first alarm in the above period and the release time. In reality, the data of each day is processed tomorrow of that. Therefore, is not possible to detect event on the day of release. Thus, the optimum detection is tomorrow of the release (detection delay $=1$ ). This one day delay is also considered in CityBN simulation. Figure 6 demonstrates that how we define false alarms and detection delay. If we signal an alarm in a period of 14 days after release it is marked as true alarm and if we signal an alarm before or after this period, it is marked as a false alarm. Detection delay is also specified in the figure as numbers in the plates. If we signal an alarm tomorrow of the release, we get only one-day delay which is the optimum condition. For any alarm after this period we define detection delay equal to 14 (as [48]).

The outputs of both WSARE and EigenEvent are $p$-values indicating the statistical significance of recent data. Depending on the desired confidence level, we may signal an alarm. For instance, given a threshold as 0.05 we signal an alarm if the $p$-value corresponding to the recent data goes lower than 0.05 . To assess the algorithms performances we use variable $p$-value threshold from 0.020 to 0.250 with the step of 0.001 (totally $231 p$-values). Each data set has temporal size of 730 days. We use the first 365 days for training the primary baseline and the next 365 days for evaluation of the algorithms. Baseline set is also incrementally updated whenever a new window arrives after day 365 . Note that agent release in all 100 data sets occurs in the second year and is guaranteed that the first year do not contain any release. A sliding window moves across the data from day 366 to day 730 and match each window with the baseline. If the match outputs a $p$-value below the threshold, then an alarm is raised. After we reached to day 730, we compute the number of false alarms and detection delays. We finally average the detection delay and false alarms for all 100 data sets and plot the AMOC Curve. In the AMOC curve, the $\mathrm{x}$-axis indicates the number of false alarms per month and the $\mathrm{y}$-axis measures the detection time in days. The optimal detection is one day detection delay with zero false alarm. The closer to the point $(0$, 1) the better detection algorithm is.

The results are shown in Fig. 7. Although the curve corresponding EigenEvent seems different comparing WSARE, if we rotate the AMOC curve 90 degrees anticlockwise we observe the same pattern similar to WSARE 3.0. The difference is that EigenEvent performs better in terms of false alarm rate and performs worse in terms of detection delay. The intersection between the curves makes the overall comparison difficult. For instance, in a desired false positive rate from 2.8 to 3.3, EigenEvent is the best method both in terms of false alarm rate and detection delay. Nevertheless, to specify which of the algorithms are better in overall we need to compute the area under the AMOC curve [34], average delay and average false positive rate (see Table 2). Obtained area under AMOC curve implies that EigenEvent outperforms all versions of WSARE. Its average false positive is considerably lower than all versions of WSARE. However, in terms of detection delay as was expected presents one more day delay. To have 
Table 2

False positive rate (per month), detection delay (in days), Area under AMOC curve and Runtime (in seconds) averaged for 100 data sets of CityBN

\begin{tabular}{lccc}
\hline Method & False positive & Detection delay & AUAMOC \\
\hline WSARE 2.0 & 4.052439 & 2.163983 & 12.859000 \\
WSARE 2.5 & 2.739062 & 2.192338 & 9.885192 \\
WSARE 3.0 & 2.877031 & $\mathbf{1 . 9 2 9 1 3 4}$ & 8.648379 \\
EigenEvent & $\mathbf{1 . 8 6 6 4 3 9}$ & 2.839827 & $\mathbf{8 . 0 2 7 8 4 2}$ \\
\hline
\end{tabular}

Table 3

Runtime (in seconds) averaged for 100 data sets

\begin{tabular}{lr}
\hline Method & Runtime \\
\hline WSARE 2.0 & 59.2 \\
WSARE 2.5 & 105.3 \\
WSARE 3.0 & 838.4 \\
EigenEvent & $\mathbf{1 6 . 8}$ \\
\hline
\end{tabular}

a separate look on both numbers of false alarms and detection delay, we also compute the average false alarms and detection delay for $231 p$-values (from 0.020 to 0.250 with the step of 0.001 ). The results are presented in Tables 4 and 5 respectively. As it can be seen from the first table, EigenEvent in terms of false alarms, beats other methods in the majority of data sets. Regarding the detection delay even though is not the best, has detected events tomorrow of release in half of the data sets.

The main reason for the differences in the performance is related to the methodological differences between EigenEvent and WSARE. EigenEvent opposed to WSARE is not a bottom up approach and subsequently is less sensitive to the small-scale changes and subsequently, presents less false positive rate. EigenEvent due to its less sensitivity to the small-scale changes reacts slower to the events. However, EigenEvent have this ability to track changes in the dimensions, for this reason does not suffer from the high false alarm rate problem of bottom-up approaches and heavy delay problem of the top-bottom approaches.

\subsection{Runtime}

Since in syndromic surveillance systems, data are often required to be processed in daily scale, computational efficiency receives less attention. In the unlikely case where data size becomes very huge and processing of data requires run-time of more than 24 hours (the process scale) then we have to come up with computational efficiency issues. Although, computational efficiency is not the claim in this research work, runtime in Table 3 indicates the superiority of EigenEvent over all versions of WSARE. EigenEvent requires only $16.8 \mathrm{~s}$ to deliver the result. This is three times faster than WSARE 2.0, 6 times faster than WSARE 2.5 and 50 times faster than WSARE 3.0. The majority of this difference is related to two factors; WSARE exhaustively search the whole space while EigenEvent only tracks the changes in the correlation structure. The second factor is related to the method the approaches compute the $p$ value of alarms. WSARE exploits Monte Carlo simulations for computing the $p$-value while EigenEvent computes the $p$-value using statistical process control techniques which is lighter.

In each time step, Eigenevent requires to perform a tensor decomposition and a matrix decomposition. The offline tensor decomposition (OTA) [42] of the baseline tensor requires $O\left(T \prod_{i=1}^{M} n_{i}\right)$ where $T$ is the temporal size of the tensor, $M$ is the order of the tensor which in our case is equal to 3 (three dimensions of space, features and time) and $n_{i}(1 \leqslant i \leqslant M)$ is the dimensionality of the $i$ th mode (reshaped matrix in dimension $i$ ). The matrix decomposition of the recent data also requires $O\left(N^{2}\right)$ for one-rank matrix decomposition. Therefore, in each step we require $O\left(T \prod_{i=1}^{M} n_{i}\right)+O\left(N^{2}\right)$. Some approximation techniques are developed for reducing the computation time of the first term. For instance, [42] proposed three different techniques including dynamic tensor analysis (DTA), streaming tensor analysis (STA) and window-based tensor analysis (WTA) that perform tensor decomposition more efficiently with much lower computation time. For instance, DTA requires computation time of 
Table 4

Number of false alarms per year for 100 datasets averaged for $231 p$-values $(0.020,0.021, \ldots, 0.250)$

\begin{tabular}{|c|c|c|c|c|c|c|c|c|c|}
\hline Dataset & WS 2.0 & WS2.5 & WS3.0 & EigenEv & Dataset & WS 2.0 & WS 2.5 & WS3.0 & EigenEv \\
\hline 1 & 70.013 & 51.8571 & 57.8485 & 37.8398 & 51 & 54.9827 & 36.1602 & 41.013 & 24.7835 \\
\hline 2 & 0 & 0 & 0 & 0 & 52 & 89.7922 & 51.29 & 59.9481 & 51.697 \\
\hline 3 & 0.4416 & 2.2251 & 1.8182 & 1.4545 & 53 & 89.2078 & 62.1991 & 61.2727 & 46.3074 \\
\hline 4 & 70.4719 & 54.3896 & 53.5368 & 30.71 & 54 & 49.0952 & 31.7403 & 33.4762 & 10.4805 \\
\hline 5 & 69.5974 & 40.5108 & 39.5758 & 29.0823 & 55 & 47.4156 & 42.1991 & 48.3896 & 29.2381 \\
\hline 6 & 93.7792 & 59.0779 & 62.0346 & 37.7056 & 56 & 108.3766 & 73.5108 & 71.3333 & 40.5498 \\
\hline 7 & 72.5628 & 50.5584 & 62.1082 & 35.3203 & 57 & 59.6017 & 36.7489 & 38.3117 & 20.0866 \\
\hline 8 & 89.5628 & 71.039 & 79.2771 & 50.0303 & 58 & 25.1299 & 20.4589 & 22.1948 & 13.7706 \\
\hline 9 & 26.3506 & 17.684 & 18.4632 & 8.2468 & 59 & 90.4372 & 41.8658 & 41.6797 & 40.0779 \\
\hline 10 & 70.0563 & 53.8831 & 49.5584 & 34.7532 & 60 & 79.0996 & 51.2208 & 52.3074 & 50.7013 \\
\hline 11 & 89.6104 & 63.2251 & 53.2771 & 48.3636 & 61 & 70.4329 & 44.29 & 50.4286 & 29.316 \\
\hline 12 & 89.3896 & 71.3333 & 75.1342 & 48.4805 & 62 & 31.5455 & 19.1645 & 19.7879 & 9.342 \\
\hline 13 & 82.8442 & 51.0996 & 49.0346 & 40.0346 & 63 & 59.3463 & 42.632 & 45.2511 & 33.2597 \\
\hline 14 & 35.7013 & 25.2597 & 25.4762 & 17.632 & 64 & 72.6407 & 48.4892 & 51.9221 & 37.29 \\
\hline 15 & 80.7619 & 44.6926 & 43.6407 & 34.7879 & 65 & 29.2251 & 19.684 & 20.2857 & 9.2684 \\
\hline 16 & 58.8745 & 42.7056 & 42.5195 & 25.7273 & 66 & 3.2771 & 2.3723 & 3.987 & 0.8312 \\
\hline 17 & 5.5714 & 4.2208 & 6.6753 & 1.3939 & 67 & 39.8398 & 22.6537 & 22.9351 & 10.2468 \\
\hline 18 & 62.0433 & 42.0606 & 44.0346 & 16.2338 & 68 & 30.5844 & 13.4286 & 15.8052 & 7.4286 \\
\hline 19 & 55.1472 & 38.7532 & 45.6017 & 32.9567 & 69 & 9.0476 & 8.0087 & 10.5931 & 2.9004 \\
\hline 20 & 31.8225 & 31.3896 & 30.4675 & 17.3463 & 70 & 1.0952 & 0 & 0 & 0 \\
\hline 21 & 17.3074 & 9.9221 & 11.8831 & 8.3117 & 71 & 7.961 & 7.7489 & 9.5281 & 2.7186 \\
\hline 22 & 42.4502 & 22.3506 & 20.9091 & 11.1775 & 72 & 9.974 & 9.645 & 7.7835 & 2.9697 \\
\hline 23 & 10.9913 & 7.2251 & 8.6667 & 0.9524 & 73 & 35.7749 & 28.3939 & 27.0476 & 16.6667 \\
\hline 24 & 29.4199 & 20.2338 & 26.2035 & 10.1255 & 74 & 18.5801 & 13.7706 & 13.0563 & 6.3853 \\
\hline 25 & 35.1905 & 25.3377 & 22.7706 & 14.5455 & 75 & 78.0433 & 44.7316 & 56.9134 & 35.2424 \\
\hline 26 & 84.2597 & 54.7229 & 57.4589 & 50.0909 & 76 & 73.1082 & 48.8095 & 48.6753 & 52.2165 \\
\hline 27 & 9.987 & 9.8052 & 11.2641 & 4.4372 & 77 & 8.8615 & 8.2944 & 8.3983 & 0.2381 \\
\hline 28 & 20.3896 & 16.4632 & 14.7316 & 3.1732 & 78 & 29.5238 & 21.5108 & 12.5455 & 10.697 \\
\hline 29 & 0 & 0 & 0 & 0 & 79 & 76.4675 & 53.1732 & 55.961 & $\mathbf{5 0 . 9 4 3 7}$ \\
\hline 30 & 93.2468 & 65.0736 & 69.4156 & 48.4199 & 80 & 48.5152 & 23.4459 & 23.5325 & 15.3074 \\
\hline 31 & 47.8701 & 35.5108 & 36.8398 & 32.8528 & 81 & 4.2338 & 10.303 & 9.1645 & 4.5801 \\
\hline 32 & 74.7013 & 60.7835 & 70.7576 & 40.3939 & 82 & 38.316 & 22.0779 & 21.9481 & 10.3377 \\
\hline 33 & 0 & 0 & 0.6537 & $\mathbf{0}$ & 83 & 85.9004 & 57.8052 & 57.1775 & 40.6797 \\
\hline 34 & 71.7229 & 40.6623 & 40.9437 & 20.0866 & 84 & 8.3074 & 4.7965 & 4.5152 & 0.7749 \\
\hline 35 & 88.7706 & 54.7489 & 48.5152 & 52.1472 & 85 & 20.303 & 14.8095 & 12.4978 & 3.1255 \\
\hline 36 & 61.2468 & 50.4719 & 40.6407 & 39.8268 & 86 & 77.6061 & 42.0996 & 49.8745 & 35.7532 \\
\hline 37 & 31.0866 & 15.4719 & 12.961 & 16.8918 & 87 & 22.5671 & 18.0173 & 22.4459 & 8.658 \\
\hline 38 & 31.6104 & 23.2771 & 27.3247 & 18.7662 & 88 & 8.3723 & 6.5238 & 7.0303 & 1.316 \\
\hline 39 & 95.3203 & 65.1385 & 73.9913 & 46.8831 & 89 & 57.5108 & 40.0606 & 51.1212 & 21.7229 \\
\hline 40 & 33.3939 & 18.9307 & 23.9177 & 15.8874 & 90 & 67.71 & 46.0433 & 58.1385 & 24.2381 \\
\hline 41 & 65.0909 & 39.8918 & 39.2208 & 31.29 & 91 & 67.7749 & 48.6797 & 43.1169 & 20.7056 \\
\hline 42 & 77.7359 & 43.7273 & 49.8528 & 24 & 92 & 68.316 & 55.5541 & 56.2035 & 34.9221 \\
\hline 43 & 30.9264 & 20.4719 & 20.5887 & 10.4762 & 93 & 66.987 & 48.7013 & 47.1039 & 28.5022 \\
\hline 44 & 63.0303 & 46.039 & 48.8918 & 36.4416 & 94 & 28.697 & 24.1688 & 27.7056 & 9.7576 \\
\hline 45 & 81.7879 & 47.7792 & 49.3506 & 45.2771 & 95 & 69.4632 & 48.1169 & 47.2944 & 33.2814 \\
\hline 46 & 84.7749 & 65.7662 & 67.8355 & 41.4589 & 96 & 34.0216 & 16.8528 & 18.2121 & 12.4502 \\
\hline 47 & 8.3377 & 11.1688 & 9.684 & 0.5108 & 97 & 4.0996 & 6.1818 & 7.5498 & 1.619 \\
\hline 48 & 43.6234 & 19.7879 & 29.7489 & 15.4242 & 98 & 34.0476 & 18.4372 & 15.0087 & 14.7403 \\
\hline 49 & 26.5195 & 14.1861 & 18.1169 & 15.4286 & 99 & 56.0909 & 30.987 & 42.8485 & 22.7186 \\
\hline 50 & 33.4329 & 31.6797 & 35.3117 & 16.5671 & 100 & 66.7922 & 46.4242 & 50.5844 & 28.9394 \\
\hline
\end{tabular}

$$
2 \sum_{i=1}^{M} r_{i} n_{i}^{2}+\sum_{i=1}^{M} n_{i} \sum_{j=1}^{M} n_{j}
$$


Table 5

Detection Delay (in days) for 100 datasets averaged for $231 p$-values $(0.020,0.021, \ldots, 0.250)$

\begin{tabular}{|c|c|c|c|c|c|c|c|c|c|}
\hline Dataset & WS 2.0 & WS 2.5 & WS3.0 & EigenEv & Dataset & WS 2.0 & WS 2.5 & WS3.0 & EigenEv \\
\hline 1 & 1.3463 & 2 & 1.3463 & 2 & 51 & 1 & 1 & 1 & 3.5325 \\
\hline 2 & 1.0433 & 1 & 1 & 1.2987 & 52 & 1 & 1 & 1 & 2.0693 \\
\hline 3 & 2 & 2 & 2 & 12.9394 & 53 & 3.7835 & 8.7532 & 7.7706 & 4.8312 \\
\hline 4 & 1 & 1 & 1 & 1 & 54 & 1 & 1 & 1 & 1 \\
\hline 5 & 1 & 1 & 1 & 2.7273 & 55 & 1 & 1 & 1 & 1 \\
\hline 6 & 4.7056 & 6.2035 & 5.2511 & 6.0346 & 56 & 1 & 1 & 1 & 1 \\
\hline 7 & 1 & 1 & 1 & 1.5065 & 57 & 1 & 1 & 1 & 1 \\
\hline 8 & 1.2251 & 1 & 1 & 1.6104 & 58 & 1 & 1 & 1 & 1 \\
\hline 9 & 1.5628 & 1.2771 & 1.1688 & 1 & 59 & 5.0909 & 4.4892 & 6.1212 & 11.0606 \\
\hline 10 & 1 & 9.1905 & 1 & 1 & 60 & 3.039 & 2 & 1.5628 & 5.4286 \\
\hline 11 & 1 & 1 & 1 & 1.5974 & 61 & 1.9957 & 2 & 2 & 2 \\
\hline 12 & 1 & 1 & 1 & 1 & 62 & $1 \quad$ & 1 & 1 & 1 \\
\hline 13 & 4.2078 & 1 & 1 & 7.8139 & 63 & 1 & 2 & 1 & 1 \\
\hline 14 & 1 & 1 & 1 & 1 & 64 & 4.5065 & 13.9913 & 1.7316 & 2.026 \\
\hline 15 & 2.3593 & 1 & 1 & 1.7316 & 65 & 4.0779 & 7.6364 & 10.4156 & 8.4502 \\
\hline 16 & 1.039 & 1 & 1 & 1.0996 & 66 & 2 & 1.3463 & 2 & 1.5758 \\
\hline 17 & 1 & 1 & 1 & 1.5065 & 67 & 2.8095 & 1 & 1 & 1.3506 \\
\hline 18 & 1 & 1 & 1 & 1.026 & 68 & 1 & 1 & 1 & 1 \\
\hline 19 & 1.3074 & 1 & 1.2338 & 2 & 69 & 1 & 1 & 1 & 3.8139 \\
\hline 20 & 1 & 1 & 1 & 1 & 70 & 1 & 1 & 1 & 2.5931 \\
\hline 21 & 1 & 1 & 1 & 1 & 71 & 1 & 1 & 1 & 1 \\
\hline 22 & 1 & 1 & 1 & 1 & 72 & 6.5368 & 3 & 3 & 11.5455 \\
\hline 23 & 1 & 1 & 1 & 1 & 73 & 4 & 7.5758 & 6.3463 & 5.3333 \\
\hline 24 & 1 & 1 & 1 & 1 & 74 & 1.2597 & 1 & 1 & 1 \\
\hline 25 & 1 & 1 & 1 & 1 & 75 & 1 & 2.1255 & 1 & 1 \\
\hline 26 & 2.5541 & 2 & 1.2035 & 5.0649 & 76 & 1.2121 & 2 & 1 & 1.2251 \\
\hline 27 & 1.4329 & 1 & 1 & 1 & 77 & 1.9091 & 1 & 1 & 2.2468 \\
\hline 28 & 1 & 1 & 1 & 1 & 78 & 10.0779 & 7.4632 & 13.7403 & 11.2294 \\
\hline 29 & 1 & 1 & 1 & 1 & 79 & 4.2554 & 8.1039 & 4.961 & 8.7532 \\
\hline 30 & 8.6883 & 2.0823 & 2 & 4.2338 & 80 & 1 & 1 & 1 & 1 \\
\hline 31 & 1 & 1 & 1 & 1 & 81 & 1 & 1 & 1 & 1 \\
\hline 32 & 5.4242 & 1.2597 & 1 & 2.3506 & 82 & 1.4502 & 1 & 1 & 1 \\
\hline 33 & 1 & 1 & 1 & 1 & 83 & 6.6753 & 4.5022 & 7.355 & 5.6147 \\
\hline 34 & 1 & 1 & 1 & 1 & 84 & 1.3463 & 1 & 1 & 1 \\
\hline 35 & 3.6883 & 1.329 & 1.8788 & 5.6623 & 85 & 1 & 1 & 1 & 1 \\
\hline 36 & 2.2597 & 6.4935 & 7.1126 & 10.1082 & 86 & 1.7835 & 2 & 2 & 1.974 \\
\hline 37 & 1 & 1 & 1 & 1 & 87 & 2.9134 & 2.645 & 1 & 4.0823 \\
\hline 38 & 1 & 1 & 1 & 1 & 88 & 1 & 1 & 1 & 3.0823 \\
\hline 39 & 5.7186 & 6.4242 & 10.0649 & 10.7273 & 89 & 1 & 1 & 1 & 1 \\
\hline 40 & 1.0476 & 1 & 1 & 1 & 90 & 1 & 1 & 1 & 1 \\
\hline 41 & 1 & 1 & 1 & 1 & 91 & 2.1732 & 1.0866 & 1 & 7.1602 \\
\hline 42 & 1.5628 & 1 & 1 & 1 & 92 & 6.8095 & 3.4762 & 5.1429 & 11.1602 \\
\hline 43 & 1 & 10.0779 & 1 & 1 & 93 & 1.5628 & 1.3463 & 1 & 2.329 \\
\hline 44 & 2.329 & 1 & 1 & 1.961 & 94 & 1.2468 & 1.329 & 1.1299 & 1.9827 \\
\hline 45 & 6.3074 & 2.3853 & 2.039 & 1 & 95 & 1.4502 & 1 & 1 & 1 \\
\hline 46 & 4.5281 & 1 & 1 & 6.8831 & 96 & 1 & 1 & 1 & 1 \\
\hline 47 & 7.3506 & 10.2944 & 7.9913 & 12.9957 & 97 & 1 & 1 & 1 & 1 \\
\hline 48 & 1.2727 & 1 & 1 & 1.3593 & 98 & 3.645 & 1 & 1 & 1.3377 \\
\hline 49 & 4.8268 & 3.3463 & 2.3463 & 1 & 99 & 1 & 1 & 1 & 1 \\
\hline 50 & 1 & 1 & 1 & 1 & 100 & 1 & 1 & 1 & 1.9567 \\
\hline
\end{tabular}

where $r_{i}$ is the core size for each mode which in our case is equal to 1 . Therefore, assuming the tensor with three dimensions (as our case study) we require only $2 \sum_{i=1}^{3} n_{i}^{2}+\sum_{i=1}^{3} n_{i} \sum_{j=1}^{3} n_{j}$ which is a tremendous improvement over OTA. For low-order tensor (i.e. $M \leqslant 5$ ) as is pointed out in [42], the 


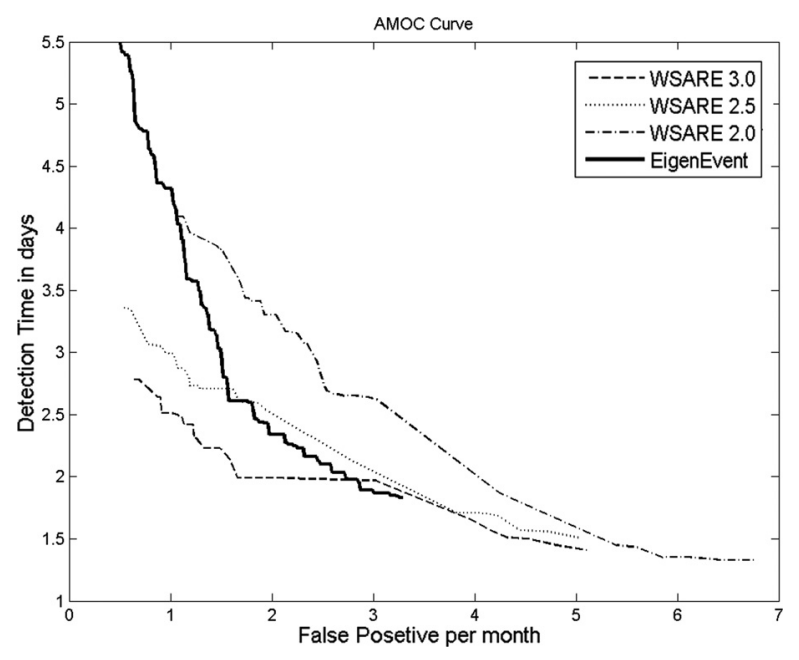

Fig. 7. AMOC curve for EigenEvent vs. WSARE.

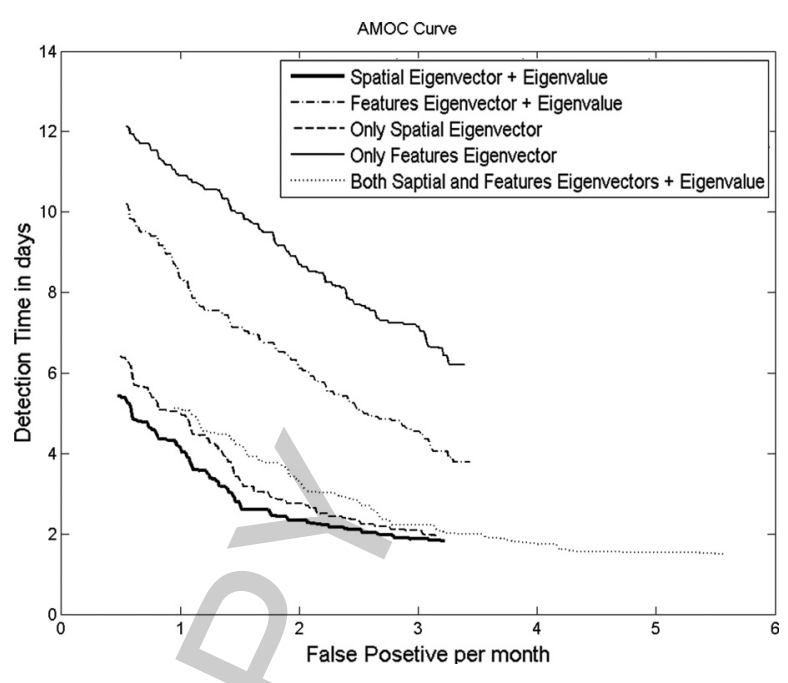

Fig. 8. Effect of indicators on the performance.

diagonalization which is the main cost can be performed via faster approximation approaches. However, since computation efficiency is not the main concern in this part of our research we do not test all the available techniques.

\subsection{Leading indicators}

As we mentioned before, EigenEvent algorithm tracks the deviation of sliding window eigenspace from the baseline tensor eigenspace for change detection. Now the question is that what elements of the eigenspace we should take for the match. Should we opt for eigenvectors corresponding to the spatial dimension or to the feature dimension. Should eigenvalue be used along eigenvector or eigenvector alone is enough. We examine five circumstances: the first condition is the default setting in the Algorithm (The optimum selection in line 9 of Algorithm 1), and the rest are different combination of eigenvectors and eigenvalues. Figure 8 illustrates the AMOC curve for these different combinations. As it can be seen, by using only spatial eigenvector (without considering eigenvalue) we experience the same result but with more half-day average delay. In fact, involving of Eigenvalue in the change detection process provides earlier detection. We also study a condition where whole eigenspace is used. In this case we take into account both spatial and features eigenvectors along the eigenvalue. This leads to half-day delay earlier detection, but with 1.5 more false alarms. Excluding spatial eigenvector from the eigenspace matching also leads to lower performance both in terms of delay and false alarms. This result reveals that how the spatial dimension is important. In fact, temporal methods that exclude the spatial dimension loose lots of information. The reason is that feature signals are very noisy and detection of pattern of such noisy data comes with high false discovery. Instead, the spatial dimension is more stable and tracking changes in this dimension can be a better indicator for tracking particular events such as disease outbreaks (our case study), because, one of the key signatures of disease outbreak is movement in the space. This movement changes the constant patterns in the spatial dimension and subsequently this appears in the principal spatial eigenvector.

\subsection{Baseline selection}

We compare three scenarios for baseline creation: 1) from historical set without respect to the environ- 


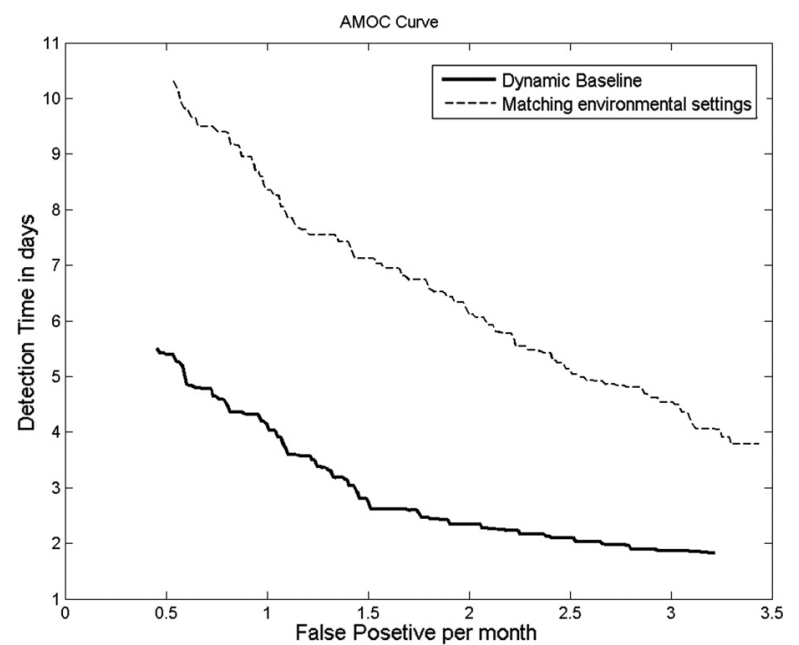

Fig. 9. Dynamic baseline vs. environmental matching baseline.

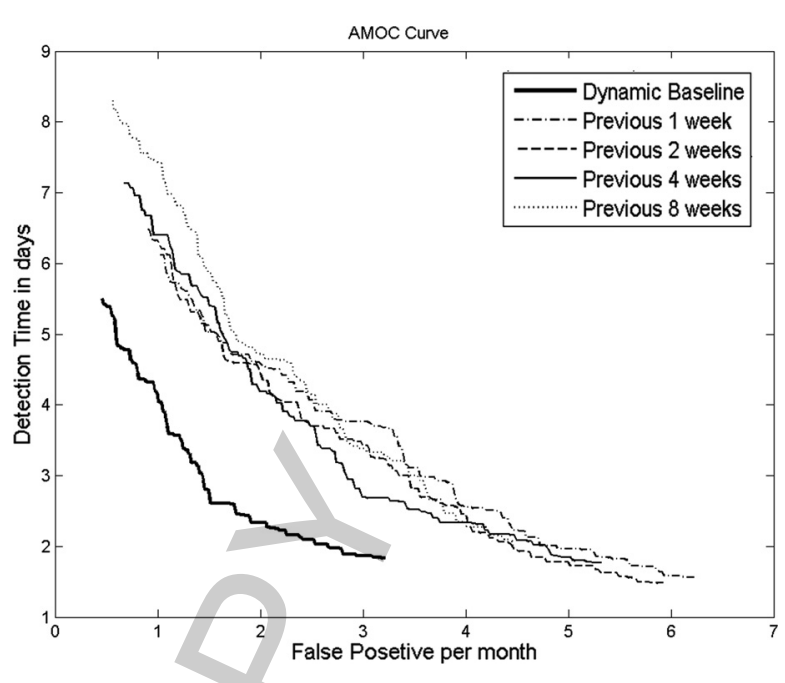

Fig. 10. Dynamic baseline vs. historical baseline.

mental setting; 2) from historical set with respect to the environmental setting; and 3) dynamic baseline tensor (our strategy). In the first scenario we compare the recent data with historical data without considering the environmental setting. For instance, we compare the recent data with data of last one week or the last eight weeks. In the second scenario we take reference data from the matched environmental setting of the day. For instance, if the environmental setting of recent day is 4112 we search in historical set for those Space $\times$ Features matrices whose corresponding environmental setting is 4112 . In the third scenario (our method), we create the baseline tensor from matched environmental setting, but we give more importance to the most dominant environmental setting and more recent data.

Figures 9 and 10 compare the obtained performance through these different strategies. Figure 10 illustrates the comparison of the first scenario versus the third scenario and Fig. 9 compares the performance of the second scenario versus the third scenario. The results reveal that our dynamic tensor creation strategy outperforms the first and second scenarios. The reason of this good performance is related to this that our approach makes a batter inference for unseen environmental setting. This approach is also robust to the noises and therefore provides a higher quality baseline reference.

\section{Conclusion and future works}

We propose a novel approach based on eigenspace techniques for early detection of events from complex data streams in syndromic surveillance. The purpose of this work is to reduce the false alarm rate of the state-of-the-art early detection methods. The experimental evaluation results on benchmark data sets shows that the proposed approach provides a better overall performance versus state-of-the-art. Our approach while maintains the detection delay in a reasonable level improves the false alarm rate to a considerable extent. While top-down approaches look for changes in higher level feature space and bottom-up approaches track changes in the low-level feature space, we introduce a novel methodology based on eigenspace and tensor decomposition techniques that track changes both in high level and the dimension level. The overall changes in the system appear in the eigenvalue and a change in the dimensions appears in the eigenvectors. Such dimension-based strategy is very helpful in some applications 
such as disease outbreak where the spatial dimension gets very important. However, using such methods makes sense when data contains further dimensions (e.g. Space and time). In other words, the competitive part of our approach is its dimension-based change tracking which is valid only for multidimensional (multiway) data.

A challenge to the future research is to utilize EigenEvent in a real-world problem and evaluate its performance in the practice. This was one of our main limitations in this research. Unfortunately, there is no public real-world data available with ground truth for syndromic surveillance research. Most of bio-surveillance programs also correspond to the governmental sections where gaining data in most of the time is impossible. Even if we access to real data, the period of outbreaks or events is not specified in that. There is a recent developed simulator [29] that simulates multivariate syndromic time series and outbreak signatures. However, since this simulator does not support the spatial dimension as the future work we are going to adapt it for this purpose and perform more experiments based on the new simulated data sets. We also intend to study the computational performance of the algorithm using incremental and streaming tensor decomposition techniques [43] which are more appropriate for large-scale data sets.

\section{Complements}

The MATLAB code and data sets are available online via http://fanaee.com/research/EigenEvent.

\section{Acknowledgments}

This research was supported by the Projects NORTE-07-0124-FEDER-000059 and NORTE-07-0124FEDER-000056 which is financed by the North Portugal Regional Operational Program (ON.2 - O Novo Norte), under the National Strategic Reference Framework (NSRF), through the European Regional Development Fund (ERDF), and by national funds, through the Portuguese funding agency, FundaçÓćo para a CiéŸŕcia ea Tecnologia (FCT). Authors also acknowledge the support of the European Commission through the project MAESTRA (Grant Number ICT-750 2013-612944).

\section{References}

[1] C.P. Barros, An intervention analysis of terrorism: The spanish eta case, Defence and Peace Economics 14(6) (2003), 401-412.

[2] S.D. Bay and M.J. Pazzani, Detecting change in categorical data: Mining contrast sets, in: Proceedings of the Fifth ACM SIGKDD International Conference on Knowledge Discovery and Data Mining, ACM (1999), 302-306.

[3] D.L. Buckeridge, H. Burkom, M. Campbell, W.R. Hogan and A.W. Moore, Algorithms for rapid outbreak detection: A research synthesis, Journal of Biomedical Informatics 38(2) (2005), 99-113.

[4] G.F. Cooper, D.H. Dash, J.D. Levander, W.-K. Wong, W.R. Hogan and M.M. Wagner, Bayesian biosurveillance of disease outbreaks, in: Proceedings of the 20th Conference on Uncertainty in Artificial Intelligence, AUAI Press (2004), 94-103.

[5] G.F. Cooper, D.H. Dash, J.D. Levander, W.-K. Wong, W.R. Hogan and M.M. Wagner, Bayesian biosurveillance of disease outbreaks, in: Proceedings of the 20th Conference on Uncertainty in Artificial intelligence UAI'04, Arlington, Virginia, United States, AUAI Press, (2004), 94-103.

[6] L. De Lathauwer, B. De Moor and J. Vandewalle, A multilinear singular value decomposition, SIAM Journal on Matrix Analysis and Applications 21(4) (2000), 1253-1278.

[7] G. Dong and J. Li, Efficient mining of emerging patterns: Discovering trends and differences, in: Proceedings of the Fifth ACM SIGKDD International Conference on Knowledge Discovery and Data Mining, ACM (1999), 43-52.

[8] Y. Dong, Y. Li and M. Lai, Structural damage detection using empirical-mode decomposition and vector autoregressive moving average model, Soil Dynamics and Earthquake Engineering 30(3) (2010), 133-145. 
[9] W. Enders and T. Sandler, The effectiveness of antiterrorism policies: A vector-autoregression-intervention analysis, American Political Science Review 87(4) (1993), 829-844.

[10] H. Fanaee-T and J. Gama, Event labeling combining ensemble detectors and background knowledge, Progress in Artificial Intelligence (2013), 1-15.

[11] T. Fawcett and F. Provost, Activity monitoring: Noticing interesting changes in behavior, in: In Proceedings of the Fifth ACM SIGKDD International Conference on Knowledge Discovery and Data Mining (1999), 53-62.

[12] P.H. Gesteland, R.M. Gardner, F.-C. Tsui, J.U. Espino, R.T. Rolfs, B.C. James, W.W. Chapman, A.W. Moore and M.M. Wagner, Automated syndromic surveillance for the 2002 winter olympics, Journal of the American Medical Informatics Association 10(6) (2003), 547-554.

[13] E. Gursky, T.V. Inglesby and T. O'toole, Anthrax 2001: Observations on the medical and public health response, Biosecurity and Bioterrorism: Biodefense Strategy, Practice, and Science 1(2) (2003), 97-110.

[14] J. Hanely and B. McNeil, The meaning and use of the area under a receiver operating characteristic (roc) curve, Radiology 143(1) (1982), 29-36.

[15] K.J. Henning, Overview of syndromic surveillance, what is syndromic surveillance, MMWR Morb Mortal Wkly Rep 53(Suppl) (2004), 5-11.

[16] M.L. Hutwagner, M.W. Thompson, G.M. Seeman and T. Treadwell, The bioterrorism preparedness and response early aberration reporting system (ears), Journal of Urban Health 80(1) (2003), i89-i96.

[17] X. Jiang and G.F. Cooper, A bayesian spatio-temporal method for disease outbreak detection, Journal of the American Medical Informatics Association 17(4) (2010), 462-471.

[18] Z. Kaufman, E. Cohen, T. Peled-Leviatan, C. Lavi, G. Aharonowitz, R. Dichtiar, M. Bromberg, O. Havkin, Y. Shalev, R. Marom et al., Using data on an influenza b outbreak to evaluate a syndromic surveillance system-israel, June 2004, MMWR (CDC) 54(Suppl) (2005), 191.

[19] V. Klema and A. Laub, The singular value decomposition: Its computation and some applications, Automatic Control, IEEE Transactions on 25(2) (1980), 164-176.

[20] T.G. Kolda and B.W. Bader, Tensor decompositions and applications, SIAM Review 51(3) (2009), 455-500.

[21] W. Ku, R.H. Storer and C. Georgakis, Disturbance detection and isolation by dynamic principal component analysis, Chemometrics and Intelligent Laboratory Systems, InCINC '94 30(1) (1995), 179-196.

[22] M. Kulldorff, A spatial scan statistic, Communications in Statistics-Theory and Methods 26(6) (1997), 1481-1496.

[23] M. Kulldorff, Spatial scan statistics: Models, calculations, and applications, in: Scan Statistics and Applications, Springer (1999), 303-322.

[24] M. Kulldorff, W. Athas, E. Feurer, B. Miller and C. Key, Evaluating cluster alarms: A space-time scan statistic and brain cancer in los alamos, new mexico, American Journal of Public Health 88(9) (1998), 1377-1380.

[25] M. Kulldorff, F. Mostashari, L. Duczmal, Y.W. Katherine, K. Kleinman and R. Platt, Multivariate scan statistics for disease surveillance, Statistics in Medicine 26(8) (2007), 1824-1833.

[26] M. Kulldorff and N. Nagarwalla, Spatial disease clusters: Detection and inference, Statistics in Medicine 14(8) (1995), 799-810.

[27] M. Kurucz, A.A. Benczúr and K. Csalogány, Methods for large scale svd with missing values, in: Proceedings of KDD Cup and Workshop, Citeseer 12 (2007), 31-38.

[28] Y. Le Strat and F. Carrat, Monitoring epidemiologic surveillance data using hidden markov models, Statistics in Medicine 18(24) (1999), 3463-3478.

[29] T. Lotze, G. Shmueli and I. Yahav, Simulating multivariate syndromic time series and outbreak signatures, Robert $H$ Smith School Research Paper No. RHS-06-054 (2007).

[30] J. MacGregor and T. Kourti, Statistical process control of multivariate processes, Control Engineering Practice 3(3) (1995), 403-414.

[31] A.W. Moore, B. Anderson K. Das and W.-K. Wong, Combining multiple signals for biosurveillance, Handbook of Biosurveillance (2006), 235.

[32] V. Moskvina and A. Zhigljavsky, An algorithm based on singular spectrum analysis for change-point detection, Communications in Statistics-Simulation and Computation 32(2) (2003), 319-352.

[33] D.B. Neill, Detection of spatial and spatio-temporal clusters, Ph D thesis, Department of Computer Science, Carnegie Mellon University, Pittsburgh, PA, USA, 2006.

[34] J. Que and F.-C. Tsui, A multi-level spatial clustering algorithm for detection of disease outbreaks, AMIA Annual Symposium Proceedings AMIA Symposium AMIA Symposium (2008), 611-615.

[35] T.M. Rath, M. Carreras and P. Sebastiani, Automated detection of influenza epidemics with hidden markov models, in: Advances in Intelligent Data Analysis V, Springer (2003), 521-532.

[36] B.Y. Reis and K.D. Mandl, Time series modeling for syndromic surveillance, BMC Medical Informatics and Decision Making 3(1) (2003), 2.

[37] R.E. Serfling, Methods for current statistical analysis of excess pneumonia-influenza deaths, Public Health Reports 78(6) (1963), 494 
[38] G. Shmueli and H. Burkom, Statistical challenges facing early outbreak detection in biosurveillance, Technometrics 52(1) (2010).

[39] G. Shmueli and S. Fienberg, Current and potential statistical methods for monitoring multiple data streams for biosurveillance, in: Statistical Methods in Counterterrorism, A. Wilson, G. Wilson and D. Olwell, eds, Springer New York, 2006, pp. 109-140.

[40] D. Siegrist and J. Pavlin, Bio-alirt biosurveillance detection algorithm evaluation, MMWR Morb Mortal Wkly Rep 53(Suppl) (2004), 152-158.

[41] K.M. Simmons and D. Sutter, False alarms, tornado warnings, and tornado casualties, Weather Climate and Society 1(1) (2009), 38-53.

[42] J. Sun, D. Tao, S. Papadimitriou, P.S. Yu and C. Faloutsos, Incremental tensor analysis: Theory and applications, $A C M$ Trans Knowl Discov Data 2(3) (Oct 2008), 11:1-11:37.

[43] J. Sun, D. Tao, S. Papadimitriou, P.S. Yu and C. Faloutsos, Incremental tensor analysis: Theory and applications, $A C M$ Transactions on Knowledge Discovery from Data (TKDD) 2(3) (2008), 11.

[44] M.M. Wagner, F. chiang Tsui, J.U. Espino, V.M. Dato, D.F. Sittig, R.A. Caruana, L.F. Mcginnis, D.W. Deerfield, M.J. Druzdzel and D.B. Fridsma, The emerging science of very early detection of disease outbreaks, in: J Pub Health Manag Pract, Addison Wesley 7 (2001), 51-59.

[45] W. Weng-Keen, A. Moore, G. Cooper and M. Wagner, Wsare datasets, (Mar 2013), http://www.autonlab.org/autonweb/ datasets/15959.html.

[46] G.D. Williamson and G.W. Hudson, A monitoring system for detecting aberrations in public health surveillance reports, Statistics in Medicine 18(23) (1999), 3283-3298.

[47] W.-K. Wong, A. Moore, G. Cooper and M. Wagner, Bayesian network anomaly pattern detection for disease outbreaks, in: Proceedings of the Twentieth International Conference on Machine Learning, Menlo Park, California, T. Fawcett and N. Mishra, eds, AAAI Press, (Aug 2003), 808-815.

[48] W.-K. Wong, A. Moore, G. Cooper and M. Wagner, What's strange about recent events (wsare): An algorithm for the early detection of disease outbreaks, J Mach Learn Res 6 (Dec 2005), 1961-1998.

[49] N. Ye, Q. Chen, S.M. Emran and S. Vilbert, Hotelling t2 multivariate profiling for anomaly detection, Proc 1st IEEE SMC Inform Assurance and Security Workshop (2000).

[50] J. Zhang, F.-C. Tsui, M.M. Wagner and W.R. Hogan, Detection of outbreaks from time series data using wavelet transform, in: AMIA Annual Symposium Proceedings, American Medical Informatics Association 2003 (2003), 748. 